\title{
Serotonin syndrome
}

\section{Charlotte Elizabeth Evans, Joseph Sebastian}

Serotonin syndrome is an under-reported and under-recognised condition that occurs on administration of selective serotonin re-uptake inhibitors alone, or in combination with other medication known to increase levels of 5-hydroxytryptamine.

This case report demonstrates signs and symptoms associated with their overdose and illustrates the importance of recognition of this syndrome to instigate appropriate treatment for the patient.

S erotonin syndrome is an iatrogenic condition resulting from an excess of intrasynaptic 5-hydoxytryptamine (5HT). It is most commonly the result of a drug interaction where two or more agents that enhance serotonergic neurotransmission by different mechanisms are administered in combination or in overdose. It can go unrecognised and may rarely occur following overdose of a single serotonergic agent, as shown in the following case.

\section{CASE REPORT}

A 38-year-old woman presented to the accident andemergency department, having taken a significant overdose of $1.8 \mathrm{~g}$ of paroxetine, $280 \mathrm{mg}$ of temezapam and $400 \mathrm{mg}$ of diphenhydramine with 4 litres of cider 2 hours previously. She was drowsy with a Glasgow Coma Scale of 10/15 (E2, V3, M5), a respiratory rate of $24 / \mathrm{min}$, a pulse rate of $70 / \mathrm{min}$ and a blood pressure of $128 / 81 \mathrm{mmHg}$. Blood gas analysis on $60 \%$ oxygen showed a $\mathrm{pH}$ of $7.36, \mathrm{PaO}_{2}$ of $9.54 \mathrm{kPa}$ and $\mathrm{PaCo}_{2}$ of $3.77 \mathrm{~Pa}$. ECG showed sinus rhythm with a prolonged QT interval. Blood investigations showed a blood glucose of $5.1 \mathrm{mmol} / \mathrm{l}$ and blood alcohol level of $1658 \mathrm{mg} / \mathrm{ml}$, but were otherwise unremarkable.

Over the next $30 \mathrm{~min}$, the patient's Glasgow Coma Scale dropped to 7 (El, V1, M5), and she showed signs of airway obstruction and was therefore intubated by means of a rapid sequence induction using a size 7.5 cuffed endotracheal tube and $200 \mathrm{mg}$ of propofol and $100 \mathrm{mg}$ of suxamethonium. Sedation was maintained using a propofol infusion.

Following transfer to the intensive care unit, she developed short episodes of tonic clonic seizures affecting all four limbs and self-terminating within a minute. The patient had a tachycardia and was hypertensive, with a systolic blood pressure $>200 \mathrm{~mm} \mathrm{Hg}$. She also looked flushed, was sweating profusely and had a persistently high fever of over $38^{\circ} \mathrm{C}$ in the absence of any obvious focus of infection. After $24 \mathrm{~h}$, and again after $48 \mathrm{~h}$, when sedation was weaned, the patient was found confused and agitated with profound shivering and occasional vomiting. Neurological examination revealed dilated pupils, increased tone in all four limbs with global hyper-reflexia, and marked clonus in both lower limbs and upgoing plantars bilaterally. Further investigations including a CT brain, blood, urine and sputum cultures, and serum creatinine kinase and urinary myoglobin were performed and were all normal.
On day three of her ITU admission, these symptoms and signs had subsided and the patient was extubated and transferred to a general ward, where she received a psychiatric assessment. She was discharged from hospital 2 days later.

\section{DISCUSSION}

The term serotonin syndrome is used to describe the constellation of symptoms observed within hours to days following the administration of drugs that elevated serotonin concentration in combination or in overdose. The drugs most frequently contributing to this condition are the combination of a monoamine oxidase inhibitor and a selective serotonin reuptake inhibitor.

The clinical features include a change of mental status, neuromuscular hyperactivity and autonomic instability. ${ }^{1}$ Mental status changes range from confusion, agitation, anxiety, delirium and hallucinations to drowsiness and coma. The neuromuscular features include myoclonus, hyper-reflexia, muscle rigidity, tremor and severe shivering. Autonomic instability is seen in approximately $50 \%$ of the patients and includes most commonly hyperthermia, diaphoresis, sinus tachycardia, hypertension or hypotension, flushing of the skin, diarrhoea and vomiting. Life-threatening acute complications include coma, seizures, rhabdomyolysis and disseminated intravascular coagulation.

There are no specific tests to confirm the diagnosis of serotonin syndrome. ${ }^{2}$ Measurement of the serum concentration of the drug(s) is unhelpful. Treatment consists of discontinuing the offending drug(s) and supportive care, including administration of intravenous fluids, benzodiazepines to control delirium, and cooling measures to treat hyperthermia. In severe cases, intubation and ventilation are warranted to control the airway and ensure oxygenation. Although it was not demonstrated conclusively, specific pharmacological agents have been advocated in the treatment of this condition, including cyproheptadine given orally ${ }^{4}$ and chlorpromazine parenterally, ${ }^{5}$ both of which are 5-HT2 bockers. Most patients improve within $24 \mathrm{~h}$, although in $40 \%$ of the patients some symptoms persist longer.

The principal differential diagnosis is the neuroleptic malignant syndrome, ${ }^{3}$ which shares similar features. The main difference is that with the neuroleptic malignant syndrome the onset of symptoms and resolution of symptoms usually takes days to weeks, the muscular rigidity is often "lead pipe", and rhabdomyolysis and metabolic acidosis are more common.

\section{CONCLUSION}

Our report illustrates a severe case of serotonin syndrome unusually resulting from the overdose of a single serotonergic agent, paroxetine. Our patient developed the classic signs and symptoms within hours of ingestion, which initially puzzled the clinicians involved. The incidence of this condition is unknown, but is likely to be under-reported because it is not recognised or is confused with neuroleptic malignant syndrome. This potentially life-threatening condition requires a heightened 
clinical awareness in order to recognise and treat the condition promptly.

\section{SUMMARY}

Serotonin syndrome is an under-reported and under-recognised condition that occurs on administration of selective serotonin re-uptake inhibitors alone or in combination with other medication known to increase levels of 5- HT. This case demonstrates the signs and symptoms associated with this condition, and illustrates the importance of recognition of this syndrome in order to instigate appropriate treatment for the patient.

The report illustrates a severe case of serotonin syndrome unusually resulting from the overdose of a single serotonergic agent, paroxetine. The patient developed the classic signs and symptoms which include changes in mental status, neuromuscular hyperactivity and autonomic instability within hours of ingestion, which initially puzzled the clinicians involved. The incidence of this condition is unknown, but is likely to be under-reported because it is not recognised or is confused with neuroleptic malignant syndrome. This potentially life-threatening condition requires a heightened clinical awareness in order to recognise and treat the condition promptly.

\section{Authors' affiliations}

Charlotte Elizabeth Evans, The Royal Melbourne Hospital Intensive Care Unit, Melbourne, Victoria, Australia

Joseph Sebastian, Queens Square Hospital for Neurology and Neurodisability, London, UK

Correspondence to: Dr C E Evans, Flat 730, The Whitehouse, 9 Belvedere Rd, London SE1 8YU, UK; thewelshlady007@hotmail.com

Accepted 11 October 2006

Informed consent was obtained for publication of the person's details in this report.

\section{REFERENCES}

1 Mills KC. Serotonin syndrome. A clinical update. Crit Care Clin 1997; 13:763-83

2 Brimes $P$, Coppin D, Schmitt L, et al. Serotonin syndrome: a brief review. CMAJ 2003;168:1439-42.

3 Mason PJ, Morris VA, Balceza TJ. Serotonin syndrome. presentation of 2 cases and review of the literature. Medicine 2000;79:201-9.

4 Graudins A, Stearman A, Chan B. Treatment of serotonin syndrome with cyproheptadine. J Emerg Med 1998;16:615-19.

5 Gillman PK. Serotonin syndrome treated with chlorpromazine. J Clin Psychopharmacol 1997; 17:128-9. 